TITRE: PISTES D'INTERVENTIONS SUR LE PLAN DU LANGAGE ET DE LA MÉTACOGNITION POUR SOUTENIR L'ÉLĖVE QUI PRÉSENTE DES DIFFICULTÉS EN LECTURE ET EN ÉCRITURE

Auteur(s): CARole Boudreau, Professeure Université de SHERBrooke

Publication: Perspectives actuelles sur l'APprentissage de LA LECTURE eT DE L'ÉCRItURE/ CONTRIBUTIONS ABOUT LEARNING TO READ AND WRITE - ACTES DU SYMPOSIUM INTERNATIONAL SUR LA LITÉRACIE À L'ÉCOLE/INTERNATIONAL SYMPOSIUM FOR EDUCATIONAL LITERACY (SILE/ISEL) 2015

PAGES: $271-289$

Directeurs: Marie-France Morin, Denis Alamargot et Carolina Gonçalves.

ÉdITEUR: LES ÉDITIONS DE L'UNIVERSITÉ DE SHERBROOKE, 2016.

ISBN: 978-2-7622-0355-4

URI: HTTP://HDL.HANDLE.NET/11143/10273

DOI: HTTPS://DOI.ORG/10.17118/11143/10273 


\section{Pistes d'interventions sur le plan du langage et de la métacognition pour soutenir l'élève qui présente des difficultés en lecture et en écriture}

Carole Boudreau, professeure Université de Sherbrooke

Résumé : Depuis le début des années 2000, le nombre d'élèves qui présentent des difficultés d'apprentissage n'a cessé d'augmenter dans les milieux scolaires québécois (MELS, 2010), et ce, malgré une diminution de l'ensemble de l'effectif scolaire (MELS, 2009). Cette situation suscite des questionnements considérant le nombre important d'études ayant eu cours depuis les quinze dernières années visant à mieux connaitre les caractéristiques des élèves qui présentent des difficultés en lecture et en écriture (Allington et Cunnigham, 2007; Sprenger-Charolles et Serniclaes, 2003), ainsi que celles portant sur les interventions efficaces en classe (Pressley, 2006; Schumm et Arguelles, 2006). Dans ce chapitre, nous présenterons une synthèse des pratiques efficaces les mieux appuyées sur le plan scientifique en matière de soutien et d'intervention aux élèves qui présentent des difficultés sur le plan de la littératie. Deux dimensions liées aux pratiques à privilégier seront particulièrement touchées, soit l'aspect linguistique et la métacognition. Une synthèse finale sous forme de questions à caractère réflexif viendra clore ce chapitre.

Mots clés : Lecture-écriture-élèves en difficulté-métacognition-langage oral

Abstract: Since the beginning of the years 2000, the number of students showing learning difficulties has been constantly increasing in Quebec schools. (MELS,2010) and this, despite a decrease of number of students (MELS,2009). This raises many questions considering the number of studies realized in the last fifteen years aiming to grasp the characteristics of students with learning difficulties in reading and writing (Allington et Cunnigham, 2007; Sprenger-Charolles et Serniclaes, 2003) and the studies on efficient interventions in class (Pressley, 2006; Schumm et Arguelles, 2006). In this chapter, we present a synthesis of efficient practices with the best scientific bases, regarding support and interventions for student presenting difficulties in literacy. Two dimensions related to the practices to be privileged will be touched, that is the language dimension and the meta-cognitive dimension. A final overview in a question form of a reflexive nature will close this chapter. 
Les élèves qui peinent à développer des compétences adéquates à lire et à écrire représentent un défi pédagogique important pour les professionnels de l'enseignement. Selon Vaughn, Wanzek, Woodruff et Linan-Thompson (2007), 20 à 30 \% des enfants connaitront, au cours de leur primaire, des difficultés à bien lire. Les nombreuses études menées au cours des quinze dernières années dans le domaine de l'éducation (Allington et Cunnigham, 2007; Sprenger-Charolles et Serniclaes, 2003) ont certes contribué à identifier les obstacles les plus importants au développement de la compétence à lire et à écrire, de même qu'à identifier les caractéristiques des élèves qui présentent des difficultés dans ces domaines d'apprentissage. Parallèlement à ces avancées scientifiques, d'autres études (Pressley, 2006; Schumm et Arguelles, 2006) ont permis de cibler des pratiques pédagogiques de haut niveau et des pratiques différenciées qui contribuent à la réussite scolaire d'une majorité d'élèves, incluant ceux à risque d’échec. Malgré ces nouvelles connaissances sur le plan de la littératie, de nombreux apprenants seront confrontés, tout au cours de leur parcours scolaire, à des défis importants à l'égard du développement de la compétence à lire et à écrire. Certains d'entre eux parviendront, en dépit de leurs difficultés, à développer des compétences adéquates en lecture et en écriture, alors que d'autres connaitront un parcours scolaire plus difficile, caractérisé par un écart croissant entre les compétences acquises et celles attendues selon leur niveau d'âge (Allington, 2013). Même si de nombreuses recherches s'avèrent encore nécessaires pour mieux documenter les interventions à privilégier auprès des élèves qui présentent des difficultés en lecture et en écriture, un certain nombre de ces recherches ont permis d'identifier des pratiques favorables à la réussite de ces élèves. Par ailleurs, bien avant d’intégrer des pratiques spécifiques pour les élèves qui présentent des difficultés scolaires, le milieu de la recherche considère qu'un enseignement de haut niveau destiné à l'ensemble de la classe doit avoir préséance (Allington et Johnston, 2001; Darling-Hammond, Chung et Frelow, 2002; Paris, Paris et Carpenter, 2001; Scanlon, Gelzheiser, Vellutino, Schatschneider et Sweeney, 2010). Les pratiques de qualité, reconnues par la recherche, contribueront à réduire le nombre d'apprenants qui ne parviennent pas - ou difficilement - à développer des compétences adéquates en lecture et en écriture (Ehri, 2004; Jitendra, Edwards, Sacks et Jacobson, 2004; Foorman, Francis, Fletcher, Mehta et Schatschneider, 1998). C'est dans le but d'aider le titulaire de classe et l'enseignant spécialisé à mettre en place des interventions adéquates visant à soutenir la réussite du plus grand nombre d'élèves en difficulté sur le plan de la lecture et de l'écriture que nous avons élaboré ce chapitre, en accordant une attention plus spécifique aux habiletés langagières et métacognitives.

La première partie de ce chapitre abordera brièvement les principales caractéristiques des difficultés les plus fréquemment observées sur le plan de la lecture et de l'écriture, de même que certaines pratiques reconnues efficaces par la recherche en contexte d'enseignement aux élèves à risque d'échec scolaire. Par la suite, nous présenterons les interventions pouvant le mieux contribuer à soutenir les élèves qui présentent des difficultés dans le développement de leurs compétences à lire et à écrire, en se penchant plus particulièrement sur les dimensions langagière et métacognitive, indissociables du développement de la compétence à lire et à écrire. Une synthèse finale sous forme de questions 
visant la réflexion à l'égard des aspects langagiers et métacognitifs en contexte d'enseignement de la lecture et de l'écriture viendra clore ce chapitre.

\section{Les difficultés les plus persistantes en lecture et en écriture}

Les recherches des dernières années ont permis de soulever la complexité liée au développement de la compétence à lire et à écrire (Écalle et Magnan, 2006 Giasson, 2011; Graham, Berninger et Abbott, 2012; Graham et Harris, 2013; Paris, 2005; Pressley 2006; Seidenberg et McClelland, 1989). Pour certains élèves dont des troubles cognitifs, sensoriels ou langagiers ne peuvent expliquer les difficultés scolaires, cette complexité est suffisamment importante pour rendre l'apprentissage de la lecture et de l'écriture plus long et ardu. Or, selon des études menées par Torgesen (2004) et Juel (1988), les élèves qui présentent une difficulté avec la lecture dès la première année du primaire maintiennent cette difficulté tout au cours de leur parcours scolaire. De manière plus alarmiste, le Conseil canadien sur l'apprentissage (2008) considère que d'ici 2031, 47 \% des personnes âgées de 16 ans et plus présenteront des habiletés en lecture et en écriture qui seront insuffisantes pour fonctionner adéquatement en société. Dans la partie qui suit, nous jetterons un regard sur ces principales difficultés qui créent un obstacle au développement de l'apprentissage de la lecture et de l'écriture.

\section{Les difficultés en lecture}

Établir un portrait type de l 'élève qui présente des difficultés en lecture s'avère difficile, voire impossible. Toutefois, les études réalisées depuis de nombreuses années indiquent que les difficultés des élèves sur le plan de l'apprentissage de la lecture touchent principalement l'identification des mots écrits et la fluidité, la compréhension, de même que les habiletés métacognitives (nous aborderons le thème de la métacognition à la fin de ce chapitre).

Le manque de fluidité lors de la lecture, l'inexactitude des mots lus, la difficulté à décoder, à donner un sens aux mots du texte et au lien qui les unit contribuent à diminuer, parfois considérablement, le niveau de compréhension (Adams, 1990; Chard, Vaughn et Tyler, 2002; Lyon et Moats, 1997; Nikolopoulour, Goulandris et Snowling, 2003; Torgesen, 2004; Wolf et Katzir-Cohen, 2001). Nous savons maintenant que les élèves qui présentent des difficultés d'apprentissage de la lecture ont tendance à présenter, dès la maternelle, un niveau peu élevé de connaissances liées aux lettres, peu d'habiletés sur le plan de la conscience phonologique, en particulier sur le plan phonémique, et qu'ils arrivent difficilement à transférer les habiletés développées à l'oral dans des tâches liées à l'écrit (Juel et Minden-Cupp 2000; Snow, Burns et Griffin, 1998). Or, les résultats de nombreuses recherches indiquent que ces conditions sont largement associées à la réussite en lecture (Corriveau, Goswami et Thompson, 2010; Lonigan, Anthony, Phillips, Purpura, Wilson et McQueen, 2009). 
Outre les difficultés liées au décodage, la compréhension peut également s'avérer complexe et difficile pour certains élèves, et ce, malgré une habileté à lire avec fluidité et exactitude. De nombreux chercheurs (p. ex. Allington et McGill-Franzen, 2003; McIntyre, 2011; McIntyre, Hulan et Layne, 2011) reconnaissent maintenant que des facteurs liées à l'éducation socio-familiale peuvent expliquer le fait que certains élèves peinent à bien comprendre un texte. Le manque de connaissances générales ou un faible niveau de vocabulaire accentue la difficulté que peut avoir un élève à faire des liens avec les connaissances antérieures, à saisir le sens des mots et des phrases, à faire des prédictions et des résumés, à titre d'exemples.

\section{Les difficultés en écriture}

Comparativement à l'apprentissage de la lecture, l'apprentissage de l'écriture s'avère plus long et complexe chez l'apprenant. Les difficultés rencontrées par les élèves tout au cours de leur parcours scolaire sont plus diversifiées qu'en lecture, plus étroitement liées aux habiletés linguistiques à l'oral et plus tributaires de la culture générale et de l'éducation (Berninger et al., 2006; MacArthur, Graham et Fitzgerald, 2006). Ces difficultés touchent particulièrement le geste graphomoteur, les erreurs d'orthographe lexicale et grammaticale, de même que les processus de planification, de rédaction et de révision (Graham et Perrin, 2007). De manière générale, les élèves qui présentent des difficultés sur le plan de l'écriture ont tendance à produire des textes courts, avec un développement peu élaboré sur le plan des idées et de la structure, et un taux élevé d'erreurs orthographiques et syntaxiques (Baker, Chard, Ketterlin-Geller, Apichatabutra et Doabler, 2009; Graham et Harris, 1997).

Que ce soit sur le plan de la lecture ou de l'écriture, plusieurs recherches ont toutefois permis d'identifier un certain nombre de pratiques qui contribuent favorablement à l'amélioration des compétences à lire et à écrire des élèves qui présentent des difficultés dans ces domaines d'apprentissage. Nous savons maintenant que l'enseignement explicite des stratégies de compréhension et de rédaction s'avère une des pratiques la plus efficace pour soutenir la réussite des élèves en difficulté (Bakken, Mastropieri et Scruggs, 1997; Berkeley, Mastropieri et Scruggs, 2011; Gersten, Fuchs, Williams et Baker, 2001; National Reading Panel, 2000). La figure 1 présente un aperçu des pratiques gagnantes issues de ces études. 


\begin{tabular}{|l|}
\hline Enseignement systématique et explicite qui incorpore du modelage ainsi que des pratiques guidées et autonomes \\
\hline Rétroaction immédiate et explicite aux élèves \\
\hline Intention pédagogique clairement exprimée aux élèves \\
\hline Démonstration explicite sur l'importance d'utiliser la stratégie visée \\
\hline Mise en place d'une procédure visant à mesurer les progrès des élèves \\
\hline Encouragement à l'auto-questionnement \\
\hline
\end{tabular}

Figure 1. Exemples de pratiques gagnantes favorisant l'apprentissage de la lecture et de l'écriture

D’autres recherches (Ciullo, Lo, Wanzek et Reed, 2014; Kaldenberg, Watt et Therrien, 2014; Kang, McKenna, Arden et Ciullo, 2015) ont révélé que l'exposition fréquente à une littérature jeunesse, le recours aux stratégies métacognitives (ex : questionnement, rappel du récit, résumé, utilisation de carte conceptuelle) de même qu'à la lecture interactive contribuent positivement à l'amélioration de la compréhension. Selon le National Reading Panel (2000), l'enseignement de la lecture aux élèves qui présentent des difficultés doit être explicite, déployé de manière intensive et accorder une importance à l'aspect affectif dans la relation enseignant-élève. De plus, le temps dédié à des séances individuelles de lecture, de même qu'une rétroaction régulière à ceux qui présentent des difficultés contribueraient à améliorer la fluidité et l'exactitude des mots lus (Pressley, Roehrig, Raphael, Dolezal, Bohn, Mohan, Wharton-McDonald et Bogner, 2003; Allington, 2013). Concernant les interventions en écriture, l'enseignement explicite des stratégies de planification, de révision, l'annonce d'une intention pédagogique claire contribueraient à une amélioration de la compétence à écrire auprès des élèves qui présentent des difficultés (Graham et Harris, 2009; Gillepsie et Graham, 2014). Tant en lecture qu'en écriture, une revue de littérature menée par Kang et al., (2015) a révélé que le modelage des stratégies d'auto-régulation, la pratique guidée et un temps de pratique fréquent contribuent positivement à l'amélioration des compétences à lire et à écrire.

Par ailleurs, bien que les pratiques de qualité soient maintenant reconnues comme un facteur contribuant à la réussite des élèves sur le plan de la lecture et de l'écriture, pour certains d'entre eux qui connaitront des difficultés importantes à développer la compétence à lire et à écrire, un regard pédagogique sur des dimensions plus spécifiques telles que les habiletés langagières à l'oral ou celles liées à la métacognition doit être considéré. Ces deux dimensions, souvent négligées en contexte d'intervention auprès des élèves en difficulté, feront l'objet de la prochaine section de ce chapitre. 


\section{Les interventions les plus efficaces sur le plan linguistique et mé- tacognitif pour soutenir la réussite en lecture et en écriture des élèves qui présentent des difficultés}

Pour une majorité d'élèves, le fait d'être soumis à un enseignement de haut niveau, qui tient compte des résultats de recherche sur les meilleures interventions pédagogiques à mettre en place, contribue à leur réussite scolaire. Pour un certain nombre d'élèves, toutefois, ces pratiques à elles seules ne sont pas suffisantes et doivent être accompagnées d'interventions plus spécifiques pour permettre une meilleure réussite dans le développement de la compétence à lire et à écrire. En considérant les dimensions linguistiques et métacognitives en lien avec l'apprentissage de la lecture et de l'écriture, nous consacrerons la seconde partie du chapitre aux interventions à considérer auprès des élèves qui présentent des difficultés.

\section{La dimension linguistique}

Le développement de la compétence à lire et à écrire ne peut s'actualiser indépendamment des connaissances et des habiletés linguistiques qui se développent et se raffinent tout au cours de la vie (Berninger et al., 2006; McArthur et al., 2006). Le taux de prévalence des difficultés en lecture serait plus élevé chez les enfants ayant des difficultés langagières sévères que chez la population en général (McArthur et al., 2000). Une étude menée par Cain (2003) a démontré que les élèves qui présentent des difficultés en lecture ont aussi des difficultés à répondre adéquatement à des tâches verbales. Snowling, Gallagher et Frith (2003) ont constaté, auprès d'une population de 56 élèves potentiellement à risque d'un diagnostic de dyslexie, que ceux de 6 ans qui possédaient un bon bagage lexical, qui s'exprimaient correctement à l'oral et qui démontraient de bonnes habiletés sur le plan grammatical, avaient davantage tendance à composer pour leurs faiblesses phonologiques comparativement aux enfants qui présentaient de faibles habiletés langagières. Catts, Fey et Tomblin (1997) ont constaté, quant à eux, que $50 \%$ des lecteurs faibles présentent aussi des difficultés langagières. Les liens observés entre les difficultés langagières et les faiblesses en lecture ont d'ailleurs amené Lindsay et Dockrell (2004) à poser la question suivante: "Which kinds of language difficulties lead to particular patterns of reading difficulties? ». Sans chercher à répondre à cette question, nous exposerons, dans la partie suivante, des interventions liées à trois composantes pouvant influencer les habiletés langagières et, par le fait même, l'apprentissage de la lecture et de l'écriture, soit le vocabulaire, la morphosyntaxe et les connaissances générales.

\section{Le vocabulaire}

Le bagage lexical d'un élève contribue autant à une compréhension des textes lus, qu'à la rédaction d'un texte. À l'inverse, les élèves ayant un faible niveau de vocabulaire peuvent présenter des difficultés à comprendre plus finement un texte (Graves et Watts-Taffe, 2002; Perfetti, Landi et Oakhill, 
2005; Verhoeven, van Leeuwe et Vermeer, 2011; Spira, Bracken et Fischel, 2005; Stanovich, 2002). Or l'acquisition de mots nouveaux peut s'avérer complexe et longue pour un bon nombre d'élèves, notamment en ce qui concerne les mots de relation qui jouent un rôle déterminant à l'égard d'une compréhension fine de la phrase. Pour ces élèves, le développement lexical serait favorisé par des pratiques de communications riches entre l'apprenant et l'enseignant (Mclntyre, Hulan et Layne, 2011). Une approche telle que le dialogue pédagogique développée par Goldenberg (1993) pourrait contribuer à une meilleure intégration d'un vocabulaire varié et précis. Un certain nombre d'études menées par Beck et McKeown (2007), Blachowicz et Fisher (2000), Collins (2010), Graved et Watts-Taffe (2002) et Nagy et Scott (2000) ont ainsi révélé un lien significatif entre la présence d'un environnement scolaire riche sur le plan de la littérature jeunesse, d'activités pédagogiques faisant appel au langage oral et écrit, et la présence d'un enseignant qui n’hésite pas à saisir les occasions de discuter du sens des mots et des contextes d'utilisation, et le développement du vocabulaire chez l'élève. Selon ces chercheurs, le fait de soumettre les élèves à des explications sémantiques précises, des discussions fréquentes concernant le sens des mots, de même qu'à une variété de contextes d'utilisation des mots nouveaux contribue à une meilleure rétention du vocabulaire. Ceci est particulièrement important pour les mots de relation, plus difficiles à illustrer de manière concrète (Minskoff, 2005). Pour un enseignement efficace du vocabulaire, Mclntyre et ses collaboratrices (2011) proposent une série de principes à prendre en considération. La figure 2 présente une adaptation de ces principes.

\begin{tabular}{|l|}
\hline Lorsque cela s'avère possible, permettre aux élèves de sélectionner des catégories de mots qu'ils souhaitent connaitre \\
\hline Exposer les élèves à un environnement littéraire riche \\
\hline S'assurer d'une exposition répétée des mots dans des contextes variés \\
\hline Utiliser une carte conceptuelle illustrant les mots de vocabulaire et les liens entre eux (si possible) \\
\hline Encourager une lecture riche et variée \\
\hline Encourager les discussions autour des mots de vocabulaire \\
\hline Varier les approches multimédia si possible \\
\hline Enseigner les différences entre le langage courant à l'oral et le langage littéraire \\
\hline Apprendre l'utilisation du dictionnaire et des ouvrages de référence aux élèves \\
\hline Enseigner l'utilisation des informations spécifiques à la connaissance des mots à partir des suffixes, préfixes, racines des mots, etc. \\
\hline Enseigner la manière d'avoir recours au contexte pour connaitre le sens des mots \\
\hline Modéliser une attitude de « curiosité » à l'égard du sens des mots \\
\hline Combiner une immersion linguistique riche et un enseignement explicite du vocabulaire \\
\hline
\end{tabular}

Figure 2. Principes associés à l'enseignement du vocabulaire. Adaptation de Mclntyre, Hulan et Layne (2011, p. 139) 
Les études ayant porté sur le vocabulaire montrent toutefois clairement que certaines des interventions doivent se faire le plus tôt possible auprès des élèves qui présentent un faible niveau lexical, idéalement avant l'entrée formelle en $1^{\text {re }}$ année du primaire (Biemiller, 2006; Japel, Vuattoux, Dion et Simmons, 2009). Au-delà de cette période, l'écart entre le niveau de vocabulaire des élèves et celui utilisé dans les matériels didactiques et de littérature jeunesse est plus difficile à atténuer.

\section{La morphosyntaxe}

Parce que la syntaxe utilisée à l'écrit est souvent différente de celle utilisée à l'oral, certains élèves présenteront des difficultés à donner un sens aux phrases lues ou à exprimer convenablement leur pensée en situation d'écriture. La longueur et le type de phrases, l'ordre des mots utilisés, la relation entre les mots à l'intérieur de la phrase (notamment par l'usage des mots de relation) constituent des éléments qui influencent le sens de la phrase (Minskoff, 2005). Même si le langage oral n'est pas directement un reflet du langage écrit, les liens entre ces deux formes d'expression ont été largement documentés. Selon une étude réalisée par Salus et Flood, (2003), les élèves qui évoluent dans un environnement scolaire où les interactions verbales sont permises tout en étant encadrées par l'enseignant améliorent l'expression de leur pensée en cherchant à utiliser un vocabulaire et une syntaxe appropriés. Une pratique telle que la dictée à l'adulte chez les jeunes apprenants pourrait également faciliter le passage entre l'oral et l'écrit (Balslev et Saada-Robert, 2004). Ainsi, l'accompagnement réflexif par l'enseignant sur la production verbale de l'élève transformée en production écrite permettrait une rétroaction sur différentes composantes langagières, notamment la structure de la phrase.

\section{Les connaissances générales}

La culture générale de l'élève constitue un autre élément qui influence la compétence de l'élève sur le plan de la lecture et de l'écriture. Les connaissances antérieures détenues par l'apprenant permettent un accès à de nouveaux apprentissages et, pour certains élèves en difficulté, ce manque de connaissances rend plus difficile l'ajout de nouvelles. Les élèves ayant eu peu d'expérience liée à la littératie se retrouvent souvent devant un écart important face aux apprentissages scolaires prévus (Katz, Sax et Fisher, 1998). En intégrant des discussions entre l'enseignant et les élèves à partir des activités scolaires prévues en classe et en donnant des explications supplémentaires sur des notions ou concepts méconnus par ceux en difficulté, il semble possible d'améliorer leurs connaissances et leur culture générales. L'utilisation de la lecture interactive en classe s'avère également un moyen efficace de parfaire les connaissances des jeunes apprenants. Des études réalisées par Pentimonti, Zucker, Justice et Kaderavek (2010) ont montré que ce type d'activités permet aux élèves d'acquérir de nouveaux concepts et un vocabulaire plus abstrait, stimule leur intérêt et leur curiosité, ce qui leur permet de faire des inférences et des liens avec leurs connaissances antérieures. 
Après ce bref survol des interventions à privilégier pour combler certaines lacunes langagières présentes chez des élèves dont la source des difficultés à lire et à écrire pourrait y être associée, nous abordons, dans la section suivante, le domaine de la métacognition, dont les habiletés et les connaissances qui lui sont associées permettent de distinguer les faibles lecteurs-scripteurs des lecteurs-scripteurs plus compétents.

\section{La métacognition}

La métacognition a été définie par Flavell (1987) comme étant la réflexion sur ses propres processus cognitifs à l'égard d'une tâche. Le concept de métacognition se décline habituellement sous deux angles : la conscience métacognitive et le contrôle de l'activité (monitoring) (Falardeau, Pelletier, Pelletier et Gagné, 2014). Même si la recherche dans le domaine de la métacognition est moins abondante que dans d'autres domaines liés à la littératie, plusieurs études ont contribué à mieux comprendre l'apport des connaissances et habiletés métacognitives dans le développement de la compétence à lire et à écrire. Ainsi, une étude réalisée par Lewis (2007) a montré que les connaissances et habiletés métacognitives étaient corrélées avec le niveau de compétence en lecture, notamment parce qu'elles permettent à l'élève de juger du niveau de compréhension d'un texte ou de la qualité d'une production écrite. Dans le domaine de l'écriture, Graham et Harris (2000), de même que Torrance (2007), considèrent que le niveau de contrôle métacognitif (auto-régulation) influence le développement de la compétence à écrire. De leur côté, Afflerbach et Meuwissen (2005) soulignent que la capacité à identifier les bris de compréhension et à sélectionner des stratégies plus efficaces caractériserait les bons lecteurs. Plus près de nous, une étude menée au Québec par Falardeau, Pelletier, Pelletier et Gagné (2014) auprès d'élèves du secondaire qui présentent des difficultés en lecture a révélé que ce sont particulièrement ces deux habiletés que les élèves n'arrivent pas à démontrer.

À ce jour, on trouve davantage d'études ayant cherché à comprendre la métacognition et son rôle dans le développement de compétences liées à la lecture et à l'écriture que d'études ayant porté sur des pratiques favorables à son développement, en particulier auprès des élèves qui présentent des difficultés. Les quelques recherches à cet égard ont tout de même révélé que des interventions spécifiques visant le développement des habiletés métacognitives auprès d'apprenants en difficulté contribuent à une meilleure réussite scolaire (Mason, 2004; Reis, McGuire et Neu, 2000; Trainin et Swanson, 2005). En situation d'écriture, d'autres études ont soulevé le fait que des périodes réservées aux discussions entre les élèves, de même qu'entre l'enseignant et les élèves, avant la démarche rédactionnelle, peut contribuer à améliorer la compétence à écrire un texte (Berninger, Vaughan, Abbott, Begay, Coleman, Curtin, Hawkins et Graham, 2002).

De manière plus générale, les quelques études (Edmonds, 2000; Jitendra, Hoppes et Xin, 2000; Montague, Warger et Morgan, 2000; Richie, 2005) visant à mieux documenter les pratiques favorables au développement de stratégies métacognitives et aux connaissances qui lui sont associées auprès des élèves en difficulté suggèrent une approche procédurale à partir d'une liste de rappel sous forme 
de questions à soumettre aux élèves. Selon ces études ayant expérimenté de telles approches, ces processus permettraient aux élèves en difficulté d'augmenter leurs connaissances des stratégies métacognitives, d'améliorer leurs propres processus métacognitifs et les encourageraient à devenir des apprenants autonomes. La figure 3 présente des exemples (adaptation des exemples de Richie, 2005) de questions soumises à l'élève durant une tâche scolaire.

\begin{tabular}{|l|}
\hline Dans ce paragraphe (ou ce texte), comment fais-tu pour identifier... (ex : le personnage, l'action, l'endroit, les événements, etc.) \\
\hline Que dois-tu faire en premier? \\
\hline Quelle est la prochaine étape? \\
\hline Comment fais-tu pour te souvenir de la tâche demandée? \\
\hline Comment sais-tu que cette étape est bien réalisée? \\
\hline Comment sais-tu que le travail est terminé? \\
\hline
\end{tabular}

Figure 3. Exemples de questions visant le développement d'habiletés métacognitives peu importe la situation pédagogique

Dans le domaine de l'écriture, l'approche SRSD (Self-Regulated Strategy Development), développée par Harris en 1982, et qui a fait l'objet de plusieurs études et d'une méta-analyse (Graham, Harris et McKeown, 2013), semble contribuer positivement à l'amélioration des compétences à écrire. Cette approche mise sur une participation active de l'apprenant, une rétroaction de l'enseignant adaptée aux besoins de l'élève, et un passage progressif des réflexions allant de l'enseignant vers l'élève. Le SRSD se déploie en six étapes : 1) développement et activation des connaissances antérieures; 2) discussion au sujet des stratégies à mettre en place; 3) modelage de la part de l'enseignant; 4) stratégies de mémorisation; 5) accompagnement dans l'application des stratégies et connaissances et 6) pratiques individuelles des stratégies (Graham et Masson, 2008).

Puisque les habiletés et connaissances métacognitives sont généralement plus faibles chez les élèves qui présentent des difficultés sur le plan de la lecture et de l'écriture, le développement de la conscience métacognitive à l'égard des processus d'apprentissage est reconnu comme un facteur influençant favorablement l'amélioration des compétences. 


\section{Conclusion}

Que ce soit dans des tâches d'écriture ou de lecture, l'apprenant doit continuellement recourir à un ensemble de connaissances et habiletés langagières, notamment celles associées au vocabulaire, à la morphosyntaxe, de même qu'aux connaissances générales. Les compétences langagières que développera l'élève au cours de son enfance et durant son parcours scolaire influenceront son rapport à l'écrit de façon générale. Il en va de même des connaissances et compétences liées à la métacognition. L'élève qui aura développé de bonnes stratégies à l'égard des tâches de lecture et d'écriture et qui fera preuve d'auto-régulation parviendra plus facilement à réaliser les activités demandées.

Pour clore ce chapitre, nous vous soumettons quelques questions de réflexion à l'égard de vos propres pratiques d'enseignement dans les deux dimensions traitées, soit le langage et la métacognition.

- Dans quelle mesure mes pratiques d'enseignement tiennent-elles compte des compétences langagières des élèves à l'oral, en particulier ceux qui présentent des difficultés en lecture et en écriture?

- Dans mon milieu scolaire, quelle place accorde-t-on au développement des compétences langagières des élèves, tant sur le plan expressif que réceptif?

- Quelle valeur ai-je tendance à accorder aux connaissances et stratégies métacognitives des élèves qui présentent des difficultés à lire et à écrire? 


\section{Bibliographie}

Adams, M. (1990). Beginning to read: Thinking and learning about print. Cambridge, MA: MIT Press.

Afflerbach, P. et Meuwissen, K. (2005). Teaching and Learning Self-Assessment Strategies in Middle School. Dans S. E. Israel, C. Collins Block, K. L. Bauserman et K. Kinnucan-Welsch (dir.), Metacognition in Literacy Learning. Theory, Assessment, Instruction, and Professionnal Development (p. 141-164). Mahwah, NJ: Lawrence Erlbaum Associates.

Allington, R. (2013). What really matters when working with struggling readers. Reading Teacher, 66(7), 4-14.

Allington, R. et Cunnigham (2007). School that work. Where all children read and write. $3^{\text {rd }}$ edition. Boston, MA : Pearson.

Allington, R. et Johnston, P. H. (2001). Schools that work (2nd ed). New-York : Longman.

Allington, R. et McGill-Franzen, A. (2003). The impact of summer setback on the reading achievement gap. Phi Delta Kappan, 85(2), 68-75.

Baker, S., Chard, D., Ketterlin-Geller, L., Apichatabutra, C. et Doabler, C. (2009). Teaching writing to as-risk students: The quality of evidence for self-regulated strategy development. Exceptional children, 75(3), 303-318.

Bakken, J. P., Mastropieri, M. A. et Scruggs, T. E. (1997). Reading comprehension of expository science material and students with learning disabilities : A comparaison of strategies. The Journal of Special Education, 31(3), 300-324.

Balslev, K. et Saada-Robert, M. (2004). La Dictée à l'adulte: Une activité de résolution de problème complexe pour entrer dans l'écrit? Dans Association internationale pour la recherche en didactique du français. Le français : discipline singulière, plurielle ou transversale ? Québec, 26-28 août 2004.

Beck, L. L. et McKeown, M. (2007).Increasing young low income children's oral vocabulary repertoires through rich and focused instruction. Elementary School Journal, 107(3), 251-271.

Berkeley, S., Mastropieri, M. A. et Scruggs, T. E. (2011). Reading comprehension strategy instruction and attribution retraining for secondary students with learning and other mild disabilities. Journal of Reading Disabilities, 44(1), 18-32.

Berninger, V., Rutberg, J., Abbott, R., Garcia, N., Anderson-Youngstrom, M., Brooks, A., et al. (2006). Tier 1 and Tier 2 early intervention for handwriting and composing. Journal of School Psychology, 44, 3-30.

Biemiller, A. (2006). Vocabulary development and instruction : A prerequisite for school learning. Dans S. Neuman et D. Dickinson (dir.). Handbook of early literacy research, 2. New-York: Guilford Press, 41-51. 
Blachowicz, C. L. Z. et Fisher, P. (2000). Vocabulary instruction. Dans R. Barr, M. L. Kamil, P. Mosenthal et P. D. Pearson (dir). Handbook of reading research. (Vol. II, p. 502-523). Mahwah, NJ : Erlbaum.

Catts, H.W., Fey, M. et Tomblin, B. (1997). The language basis for reading disabilities. Paper presented at the annual meeting of the Society for the Scientific Study of Reading, Chicago.

Chard, D. J., Vaughn, S. et Tyler, B. J. (2002). A synthesis of research on effective interventions for building reading fluency with elementary students with learning disabilities. Journal of Learning Disabilities, 35(5), 386-406.

Ciullo, S., Lo, S., Wanzek, J. et Reed, D. (2014). A synthesis of research on informational text reading interventions for elementary students with learning disabilities. Journal of Learning Disabilities, 35(1). Advance online publication, doi.org : 10.1177/0022219414539566

Collins, M. F. (2010). ELL preschoolers' English vocabulary acquisition from storybook reading. Early Childhood Research Quarterly, 25, 84-97.

Conseil canadien sur l'apprentissage (2008). Lire l'avenir : Pour répondre aux besoins futurs du Canada en matière de littératie. Ottawa : www.ccl.cca.ca.

Corriveau, K. H., Goswami, U. et Thompson, J. M. (2010). Auditory processing and early literacy skills in a preschool and Kindergarten population. Journal of Learning Disabilities 43, 369-382.

Darling-Hammond, L., Chung, F. et Frelow, R. (2002). Variation in teacher preparation. How well do different pathways prepare teachers to teach? Journal of Teacher Education, 53(4) SeptemberOctober 2002, 286-302

Ecalle J., et Magnan A. (2006), Des difficultés en lecture à la dyslexie : problèmes d'évaluation et de diagnostic. Glossa, 97, 4-19

Edmonds, A. L. (2000). Cognitive credit cards: Acquiring learning strategies. Teaching Exceptional Children, 31(4), 68-73.

Erhi, L. C. (2004). Teaching phoemic awareness and phonics: An explanation of the National Reading Panel meta-analysis. Dans P. McCardle et V. Chhabra (dir). The voice of evidence in reading research (p. 153-186). Baltimore, MD : Brookes.

Falardeau, E., Pelletier, C., Pelletier, D. et Gagné, J.-C. (2014). Portrait des capacités métacognitives d'élèves québécois du secondaire dans la lecture de récits. Language and Literacy, 16(2), 69-89.

Flavell, J. H. (1987). Metacognition and cognitive monitoring. American Psychologist, 34, 906-911.

Foorman, B. R., Francis, D. J., Fletcher, J. M., Mehta, P. et Schatschneider, C. (1998). The role of instruction in learning to read: Preventing reading failure in at-risk children. Journal of Educational Psychology, 90(1), 37-55.

Gersten, R., Fuchs, L. S., Williams, J. P. et Baker, S. (2001). Teaching reading comprehension strategies to students with learning disabilities : A review of research. Review of Educational Research, $71(2), 279-320$. 
Giasson, J. (2011). La lecture. Apprentissage et difficultés. Montréal : Gaëtan Morin éditeur.

Gillespie, A. et Graham, S. (2014). A meta-analysis of writing interventions for students with learning disabilities. Exceptional Children, 80(4), 454-473.

Goldenberg, C. (1993). Instructional conversation: Promoting comprehension through discussion. The Reading Teacher, 46, 316-326.

Graham, S., Berninger, V., et Abbott, R. (2012). Are attitudes toward writing and reading separable constructs? a study with primary grade children. Reading and Writing Quarterly, 28(1), 51-69. DOI: $10.1080 / 10573569.2012 .632732$

Graham, S. et Harris, K. R. (1997). It can be taught, but it does not develop naturally: Myths and realities in writing instruction. School Psychology Review, 26, 414-424.

Graham, S. et Harris, K. R. (2000). The role of self-regulation and transcription skills in writing and writing development. Educational Psychologist, 35, 3-12.

Graham, S. et Harris, K. R. (2009). Almost 30 years of writing research: Making sense of it all with The Wrath of Khan. Learning Disabilities Research \& Practice, 24, 58-68. doi.org/10.1111/j.15405826.2009.01277x

Graham, S. et Harris, K. R. (2013). Common core state standards, writing, ans students with LD : Recommendations. Learning Disabilities Research \& Practice, 28(1), 28-37.

Graham, S., Harris, K. R. et McKeown, D. (2013). The Writing of Students with Learning Disabilitites, Meta-Analysis of Self-Regulated Strategy Development Writing Intervention Studies, and Future Directions. Dans Swanson H. L., Harris, K. R. et Graham, S. (dir.). Handbook of learning disabilities, second edition. New-York: The Guilford Press.

Graham, S. et Mason, L. H. (2008). Writing Instruction for Adolescents with Learning Disabilities: Programs of Intervention Research. Learning Disabilities. Research and Pratice, 23(2), 103-112.

Graham, S. et Perin, D. (2007). A meta-analysis of writing instruction for adolescents students. Journal of Educational Psychology, 99, 445-476.

Graves, M. F. et Watts-Taffe, S. M. (2002). The place of word consciousness in a research-basedvocabulary program. Dans A.E. Farstrup et S. J. Samuels (dir.). What research has to say about reading instruction. (p. 140-165). Newark, DE : International Reading Association.

Harris, K. R. (1982). Cognitive-Behavior Modification: Application with Exceptional Students. Focus on Exceptional Children, 15(2), 1-16.

Japel, C., Vuattoux, D., Dion É. et Simmons, D. (2009). Comment faciliter le développement du vocabulaire chez les jeunes enfants à risque? Une approche basée sur la recherche. Dans A. Charron, C. Bouchard et G. Cantin (dir.). Langage et littératie chez l'enfant en service de garde éducatif. Québec : Les Presses de l'Université du Québec, 37-53.

Jitendra, A. K., Edwards, L. J., Sacks, G. et Jacobson, L. A. (2004). What research says about vocabulary instruction for students with learning disabilities. Exceptional Children 70(3), 299-322. 
Jitendra, A. K., Hoppes, M. K. et Xin, Y. P. (2000). Enhancing main idea comprehension for students with learning problems: The role of a summarization strategy and self-monitoring instruction. The Journal of Special Education, 34(3), 127-139.

Juel, C. (1988). Learning to read and write : À longitudinal study of 54 children from first through fourth grade. Journal of Educational Psychology, 80, 437-447.

Juel, C. et Minden-Cupp, C. (2000). Center for the Improvement of Early Reading Achievement, The Reading Teacher, 53, 332-335.

Kaldenberg, E., Watt, S. et Therrien, W. (2014). Reading instruction in science for students with learning disabilities : A meta-analysis. Learning Disability Quarterly. Advance online publication. doi. org/10.1177/0731948714550204

Kang, E. Y., McKenna, J. W., Arden, S. et Ciullo, S. (2015). Integrated reading and writing interventions for students with learning disabilities : A review of the litterature. Learning Disabilities Research and Practice, 31(1), 22-33.

Katz, L. C., Sax, C. et Fisher, D. (1998). Activities for a diverse classroom: connecting student. Colorado Springs: CO: Peak Parent Center.

Lewis, J. (2007). Academic Literacy : Principles and Learning Opportunities for Adolescent Readers. Dans J. Lewis et G. Moorman (dir.), Adolescent Literacy Instruction. Policies et Promising Practices (p. 143-165). Newark, DE : International Reading Association.

Lindsay, G. et Dockrell, J. E. (2004). Whose job is it? Parents' concerns about the needs of their children with language problems. Journal of Special Education, 37, 225-236.

Lonigan, C. J., Anthony, J. L., Phillips, B. M., Purpura, D. J., Wilson, S. B. et McQueen, J. D. (2009). The nature of preschool phonological processing abilities and their relations to vocabulary, general cognitive abilities and print knowledge. Journal of Educational Psychology, 101, 345-358.

Lyon, G. R. et Moats, L. C. (1997). Critical conceptual and method- ological considerations in reading intervention research. Journal of Learning Disabilities, 30, 578-588.

MacArthur, C. A., Graham, S. et Fitzgerald, J. (2006). Handbook of writing research. New York: The Guilford Press.

Mason, L. H. (2004). Explicit self-regulated strategy development versus reciprocal questioning: Effects on expository reading comprehension among struggling readers. Journal of Educational Psychology, 96(2), 283-296.

McIntyre, E. (2011). Sociocultural perspectives on children with reading difficulties. Dans A. McGillFranzen et R. L. Allington (Eds.), Handbook of reading disability research (pp. 45-56). New York: Routledge.

McIntyre, H., Hulan, N. et Layne, V. (2011). Reading instruction for diverse classroom. Researche-based, culturally responsive practice. New-York: Guilford Press. 
Ministère de l'Éducation, du Loisir et du Sport (MELS) (2009). À la même école! Les élèves handicapés ou en difficulté d'adaptation ou d'apprentissage : évolution des effectifs et cheminement scolaire à l'école publique. Québec:Gouvernement du Québec.

Ministère de l'Éducation, du Loisir et du Sport (MELS) (2010). Rencontre des partenaires en éducation. Document d'appui à la réflexion. Rencontre sur l'intégration des élèves handicapés ou en difficultés, 25 octobre 2010. Québec: Gouvernement du Québec.

Minskoff, E. (2005). Teaching reading to struggling learners. Baltimore: Paul H. Brookes Publishing Co.

Montague, M., Warger, C., et Morgan, H. (2000). Solve it!: Strategy instruction to improve mathematical problem solving. Learning Disabilities Research and Practice, 15,110-116.

Nagy, W. E. et Scott, J. A. (2000). Vocabulary processes. Dans R. Barr, M. L. Kamil, P. Mosenthal et P. D. Pearson (dir.). Handbook of reading research. (Vol. II, p. 269-284). Mahwah, NJ : Erlbaum.

National Reading Panel. (2000). Teaching children to read: An evidence-based assessment of the scientific research literature on reading and its implications for reading instruction: Summary Report. Washington DC : National Institute of Child and Development. Disponible en ligne à http://www.nichd. nih.gov/publications/nrp/smallbook.htm

Nikolopoulos, D., Goulandris, N. et Snowling, M. J. (2003). Developmental dyslexia in Greek. Dans N. Goulandris et M. J. Snowling (dir.). Dyslexia in different countries. Cross-linguistic comparison (p. 53-57). London: Whurr Publishers Ltd.

Paris, S.G., (2005). Re-interpreting the development of reading skills. Reading Research Quaterley, 40, 184-202.

Paris, S. G., Paris, A. H. et Carpenter, R. D. (2001). Effective practices for assessing young readers. CIERA Report \#3-013

Pentimonti, J. M., Zucker, T. A., Justice, L. M. et Kaderavek, J. N. (2010). Informational text use in preschool classroom read-alouds. The Reading Teacher, 63(8), 656-665.

Perfetti, C. A., Landi, N. et Oakhill, J. (2005). The acquisition of reading comprehension skill. Dans M. J. Snowling et C. Hulme (dir.). The science of reading : A handbook (p. 227-247). Oxford, UK: Blackwell.

Pressley, M., Roehrig, A., Raphael, L., Dolezal, S., Bohn, K., Mohan, L., Wharton-McDonald, R. et Bogner, K. (2003). Teaching processes in elementary and secondary education. Dans W. M. Reynolds et G. E. Miller (dir.). Handbook of psychology : Vol.7. Éducational psychology (p. 153-175). New-York: Wiley.

Pressley, M. (2006). Reading instruction that works. The case for balanced teaching (3rd Ed). New-York : The Guilford Press.

Reis, S. M., McGuire, J. et Neu, T. W. (2000). Compensation strategies used by high-ability students with learning disabilities who succeed in college. Gifted Child Quarterly, 44, 123-134. 
Richie, G. (2005). Two interventions that enhance the metacognition of students with disabilities: cognitive cue cards and correspondence training. Kairaranga, 6(2), 25-32.

Salus, P. et Flood, J. (2003). Language: A user's guide. What we say and why. New-York: The Guilford Press.

Scanlon, D. M., Gelzheiser, L. M., Vellutino, F. R., Schatschneider, C., et Sweeney, J. M. (2010). Reducing the incidence of early reading difficulties: Professional development for classroom teachers versus direct interventions for children. In P. H. Johnston (Ed.), RTI in literacy: Responsive and comprehensive (pp. 257-291). Newark, DE: International Reading Association.

Schumm, J. S. et Arguelles, M. A. (2006). Reading assessment and instruction for all learners. New-York: Guilford Press.

Seidenberg, M. S. et McClelland, J. L. (1989). A distributed, developmental model of word recognition and naming. Psychological Review, 96, 523-568

Snow, C. E., Burns, M. S. et Griffin, P. (1998). Preventing reading difficulties in young children. Washington, DC : National Academy Press.

Snowling, M. J., Gallagher, A. et Frith, U. (2003). Family risk of dyslexia is continuous: individual differences in the precursors of reading skill. Child Development, 74, 358-73.

Sprenger-Charolles, L. et Serniclaes, W. (2003). Reliability of Phonological and Surface Subtypes in Developmental Dyslexia: A Review of Five Multiple Cases Studies. Special Issue on Language Disorders and Reading Acquisition, 10(1).

Spira, Bracken, \& Fischel (2005). The effects of oral language emergent literacy and behavior skills. Developmental Psychology, 42, 225-234.

Stanovich, K. (2002). Progress in understanding reading : Scientific foundations and new fonundations. New-York: Guilford Press.

Torgesen, J. K. (2004). Preventing early reading failure. DC : American Federation of Teachers. www.aft.org/pubs-reports/american_educator/issues/fall04/reading.htm

Torrance, M. (2007). Les processus cognitifs dans le développement de compétences en écriture. Encyclopédie du développement du langage et de l'alphabétisation. London, ON: Réseau canadien de recherche sur le langage et l'alphabétisation.

Trainin, G. et Swanson, H. L. (2005). Cognition, metacognition, and achievement of college students with learning disabilities. Learning Disability Quarterly, 28, 261-272.

Vaughn, S., Wanzek, J., Woodruff, A. L. et Linan-Thompson, S. (2007). Dans D. Haager, J. Klingner et S. Vaughn. (dir.), Evidence-based reading practices for response to intervention (p.11-27). Baltimore, MD: Paul H. Brooks Publishing Co.

Verhoeven, L., van Leeuwe, J. et Vermeer, A. (2011). Vocabulary growth and reading development across the elementary school years. Scientific Studies of Reading, 15(1), 8-25. 
Wolf, M. et Katzir-Cohen, T. (2001). Reading fluency and its intervention. Scientific Studies of Reading, 5(3), 211-239. 